\title{
A New Therapy for Vitiligo Using Fire Needles: A Systematic Review of Evidence from 3618 Subjects
}

\author{
Ying Luo, ${ }^{1}$ Wei Qian, ${ }^{2,3}$ Ting Dai $\left(\mathbb{D},{ }^{3}\right.$ Yi Ru $\mathbb{D}^{1},{ }^{1}$ Xiaoying Sun, ${ }^{3}$ Le Kuai, ${ }^{1}$ Liu Liu, ${ }^{1}$ \\ Meng Xing, ${ }^{1}$ Qi Zheng, ${ }^{1}$ Ying Zhang, ${ }^{1}$ Xi Chen, ${ }^{1}$ Huaibo Zhao, ${ }^{1}$ Bin Li $\mathbb{D},{ }^{1,3}$ and Xin Li $\mathbb{D}, 1,3$ \\ ${ }^{1}$ Department of Dermatology, Yueyang Hospital of Integrated Traditional Chinese and Western Medicine, \\ Shanghai University of Traditional Chinese Medicine, Shanghai 200437, China \\ ${ }^{2}$ Ningbo Municipal Hospital of TCM, Ningbo, Zhejiang 315012, China \\ ${ }^{3}$ Institute of Dermatology, Shanghai Academy of Traditional Chinese Medicine, Shanghai 201203, China
}

Correspondence should be addressed to Bin Li; 18930568129@163.com and Xin Li; 13661956326@163.com

Received 19 May 2020; Revised 8 August 2020; Accepted 11 August 2020; Published 27 August 2020

Academic Editor: Senthamil R. Selvan

Copyright (c) 2020 Ying Luo et al. This is an open access article distributed under the Creative Commons Attribution License, which permits unrestricted use, distribution, and reproduction in any medium, provided the original work is properly cited.

\begin{abstract}
Introduction. Fire needle therapy has been reported as an effective treatment for vitiligo. However, current clinical evidence has not been systematically evaluated. The aim of this study was to determine whether fire needle therapy is effective and safe for treating vitiligo. Methods. Seven databases were searched until October 2019 for randomized controlled trials on fire needle therapy, with and without conventional treatments, versus any type of conventional therapy for treating vitiligo. The RevMan 5.3.5 software was used to perform meta-analysis of the included studies. Results. Forty-seven trials comprising 3618 patients were included. Fire needle combined with conventional vitiligo treatments had a higher efficacy (risk ratio (RR): $1.55,95 \%$ confidence interval (CI): 1.46-1.65, $P<0.00001$ and RR: $1.41,95 \%$ CI: $1.24-1.61, P<0.00001$, respectively) and a greater effect on restoring the color of the area of the skin lesion (mean difference (MD): $3.40,95 \% \mathrm{CI}: 2.11-4.69, P<0.00001$ ), increasing the pigment point of vitiligo (MD: 0.83 , 95\% CI: 0.54-1.13, $P<0.00001$ ) and improving the cytokine level (MD: 8.10, 95\% CI: 6.94-9.27, $P<0.00001$ ) and effectual time (MD: $-4.76,95 \% \mathrm{CI}:-7.33$ to $-2.19, P=0.0003$ ) than traditional methods. Limb lesions (RR: $1.60,95 \% \mathrm{CI}$ : $1.31-1.95, P<0.00001)$ were more effectively treated when the treatments included fire needles, whereas the therapeutic effect of fire needles on either the head and neck (RR: $1.13,95 \%$ CI: $0.78-1.64, P=0.52$ ) or torso lesions (RR: $1.22,95 \%$ CI: $0.82-1.81$, $P=0.33$ ) was not significantly different compared to that without fire needles. No statistically significant differences in adverse effects (RR: $1.15,95 \%$ CI: $0.89-1.49, P=0.28$ ) and recurrence rates (RR: 0.90 , 95\% CI: $0.17-4.92, P=0.91$ ) during the follow-up period were observed between treatment with and without fire needles. Conclusions. Fire needle therapy combined with other conventional treatments is useful in treating vitiligo. Further studies with larger sample sizes should be performed to make a conclusive judgment. This trial is registered with CRD42018094918.
\end{abstract}

\section{Introduction}

Vitiligo is a frequently acquired depigmentation disease of localized or generalized skin and mucosa. A typical pathological feature of the disease is a lack of melanocytes and melanin granules, and it is characterized by whitening of lesions on the skin [1]. Approximately $1 \%$ to $2 \%$ of people globally have vitiligo [2], including both adults and children. The cause of vitiligo is still unclear, but it is considered to be related to heredity, immunity, mental and neurological causes, melanin self-destruction, and oxidative stress, among other causes [3]. Vitiligo could lead to serious loss of normal appearance, which may result in negative effects on learning, work, social interaction, and the marriage status of patients. Conventional treatments for vitiligo include topical corticosteroids, calcitonin inhibitors, phototherapy, and transplantation [4]. These treatments have severe side effects including chemical drug toxicity and phototherapy diffusion in varying degrees [5]. Consequently, there is an urgent need for new approaches to treatment.

Vitiligo is called "Baidian" or "Baibofeng" in traditional Chinese medicine (TCM), which has a long history of treatment for the disease. Fire needle therapy, recorded as 
early as the time of the Yellow Emperor (475-221 BC), has been an integral part of TCM and the acupuncture method. In the procedure, a special nontoxic stainless-steel needle is inserted into the acupoint after heating (Supplementary file 1 ), thus improving localized blood flow in the skin lesions and promoting the formation of melanin [6], which has been proved to be effective for treating vitiligo $[7,8]$. However, to our knowledge, no systematic review has evaluated the benefits of fire needle therapy in patients with vitiligo. Therefore, we conducted this systematic review to comprehensively assess the clinical efficacy and safety of this classic treatment for vitiligo, in order to develop and supplement the therapeutic approaches available.

\section{Methods}

This systematic review was performed following the Cochrane Handbook for Systematic Reviews of Interventions [9], presented under the Preferred Reporting Items for Systematic Reviews and Meta-analyses (PRISMA) guidelines (Supplementary file 2).

2.1. Inclusion Criteria. In this analysis, we included randomized controlled trials (RCTs) that used interventions of either fire needles alone or fire needles combined with conventional therapy, compared with any conventional therapy for vitiligo without fire needles. We included studies in which the pricking area was on the Ashi points (local skin lesions). The patients must have been diagnosed with vitiligo, regardless of sex, age, and ethnicity.

2.2. Outcomes. The primary outcome measured in this study was the total effectiveness rate for the duration of treatment, defined as the rate of restoration of the vitiligo lesions to normal color. This was divided into four categories: (i) cured: vitiligo had subsided with the skin color restored to normal; (ii) markedly effective: vitiligo had partially subsided or reduced, with more than $50 \%$ of the skin area restored to the normal skin color; (iii) effective: part of the vitiligo had subsided or reduced; and (iv) ineffective: vitiligo had been pigmented, regenerated, or expanded with reduction of the healing number.

If the trials used the number of patients as the unit for statistical outcomes, the effectiveness rates were calculated using the following NP formula: effectiveness rate $=$ (number of cured patients + number of markedly effective patients)/ number of total patients $\times 100 \%$. If the trials used the number of vitiligo skin lesions as the unit for statistical outcomes, the effectiveness rates were calculated by the following NVSL formula: effectiveness rate $=$ (number of cured vitiligo skin lesions + number of markedly effective vitiligo skin lesions)/number of total vitiligo skin lesions $\times 100 \%$.

The secondary outcomes included total restoration of the area's color, the total increased pigment point, changes in the cytokine level, effectual time, rate of the therapy's effectiveness in different locations, and adverse effects, as well as recurrence rates. The pigment point was defined as follows: 0 points, pure white skin lesions without any pigment production; 1 point, pale white skin with a little pigmentation; 2 points, pale brown skin lesions with a lot of pigmentation; and 3 points, skin damage with tan, basic, or near normal skin color.

2.3. Selection of Studies and Data Extraction. We searched the Cochrane Library, Excerpta Medica data BASE (EMBASE), PubMed, the China National Knowledge Infrastructure Database, Chinese Scientific Journals Full Text Database, Wanfang Data Knowledge Service Platform, and the Chinese Biomedical Literature Service System. We included papers dating from the earliest citation in the databases until October 2019. The search terms included "vitiligo" or "leukoderma," combined with "fire needle".

Two investigators (R.Y. and Z.Y.) independently screened the studies according to the inclusion criteria and extracted the information based on self-designed data-extraction templates, which included the first author, study characteristics (i.e., year, duration, setting, and design), participant characteristics (i.e., mean age, sample size, TCM syndrome, and interventions), and measured outcomes.

2.4. Risk of Bias Assessments. The Cochrane Handbook [9] was used to evaluate the methodological quality of each included study in terms of the following characteristics: random sequence generation, allocation concealment, blinding of participants and personnel, blinding of the outcome assessment, incomplete outcome data, selective reporting, and other biases. The terms "low," "unclear," and "high," respectively, referred to low, uncertain, and high risks of bias. The results were cross checked by two investigators (Z.Q. and L.L.), and the disagreements were settled by discussion between them.

2.5. Data Analyses. The RevMan 5.3.5 software (Cochrane Community, London, United Kingdom) was used for the data analyses. Dichotomous data are expressed as risk ratios (RRs) with 95\% confidence intervals (CIs). Continuous data are expressed as mean differences (MDs) with 95\% CIs. For each meta-analysis, we evaluated the statistical heterogeneity. If the trial had acceptable homogeneity $\left(P>0.1, I^{2}<50 \%\right)$, a fixedeffects model was applied; if there was statistical heterogeneity $\left(P<0.1, I^{2}>50 \%\right)$, we used a random-effect model. Clinical heterogeneity was assessed by reviewing the differences in the distribution of participants' characteristics between trials (i.e., age, sex, and disease duration).

\section{Results}

3.1. Description of Studies. After performing primary searches of the seven databases, we identified 342 potential articles. We excluded 293 as they did not conform to the inclusion criteria. Eventually, 47 trials with a total of 3618 patients [10-56] were included in this review (Figure 1). Among the included articles, eight [29-32, 46, 47, 50, 55] were unpublished master's theses. 


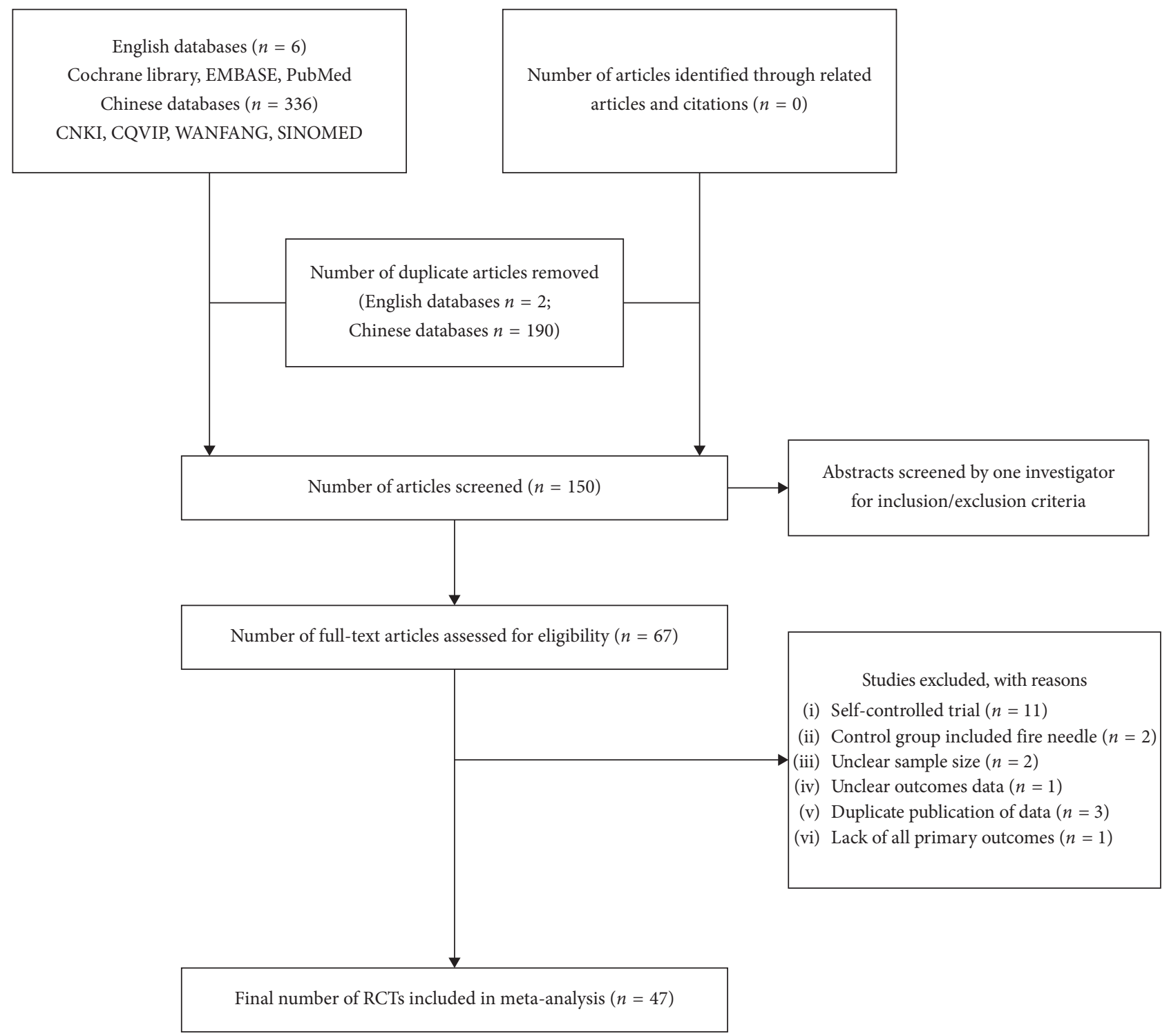

FIgUre 1: Flowchart of study selection. RCT, randomized controlled trial; EMBASE, Excerpta Medica dataBASE.

The characteristics of the included trials are presented in Table 1. All 47 trials [10-56] were performed in China. Only one trial [31] was a multicenter study, and the rest were single-center studies. The study population included children and adults. All included patients had a confirmed diagnosis of vitiligo. In the diagnosis of TCM syndromes, eight trials $[12,19,21,36,46,48,52,53]$ only included patients with "Qi-stagnancy and blood stasis," "liverkidney yin deficiency," "blockage of the vessel," or "liver depression and Qi-stagnancy" TCM patterns, as described in the TCM dialectic. Interventions included the combination of fire needle therapy on the Ashi points (local skin lesions) and conventional treatments (308-nm excimer laser, narrow-band ultraviolet B light, Western medicine ointment, and other TCM methods). The control participants used conventional treatments, without the use of fire needles. Courses of treatment ranged from 2 to 6 months.
Twelve trials [29-32, 35, 36, 42, 50, 51, 54-56] reported data on the area around the skin lesions before and after the treatments. Six trials $[32,37,52,54-56]$ reported data on the total pigment point of the skin lesions before and after the treatments. Five trials $[35,37,38,52,56]$ reported various changes of the cytokine level. Three trials $[26,36,45]$ reported the effectual time of treatments in both the experimental and control groups. Four trials $[11,21,23,26]$ examined the effect of the treatment on skin lesions placed at different locations in both the experimental and control groups. Eight trials $[14,28,31,32,39,52,54,55]$ reported follow-up data, and 41 trials [10, 11, 13-24, 26-37, 39-43, $46-53,55,56]$ reported adverse events.

3.2. Methodological Quality. The Cochrane risk of bias for all 47 trials is shown in Figure 2. Two trials [31, 32] reported using a sample-size calculation method. Eleven trials 


\begin{tabular}{|c|c|c|c|c|c|c|c|c|c|}
\hline $\begin{array}{c}3 \\
\stackrel{3}{0} \\
\overrightarrow{0}\end{array}$ & 㟔 & 觉 & 艺 & 㟔 & $n$ & 号 & 号 & 号 & 号 \\
\hline 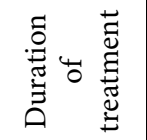 & 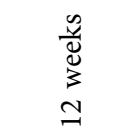 & 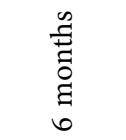 & 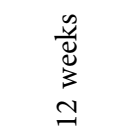 & 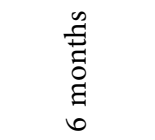 & 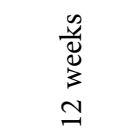 & 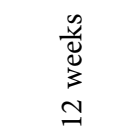 & 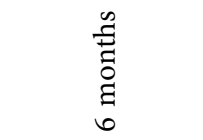 & 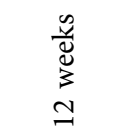 & $\begin{array}{l}\mathscr{n} \\
\mathbb{0} \\
3 \\
6 \\
-1\end{array}$ \\
\hline 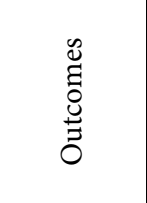 & 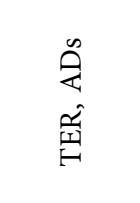 & 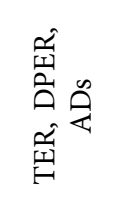 & 舀 & 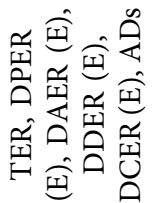 & 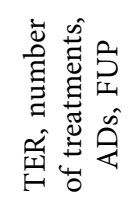 & 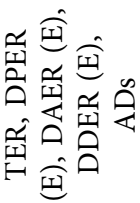 & 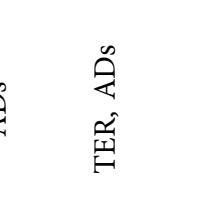 & 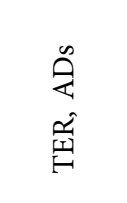 & 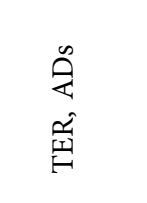 \\
\hline 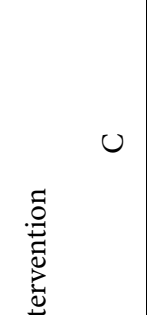 & 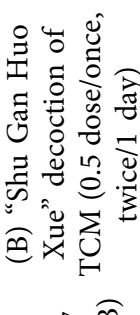 & 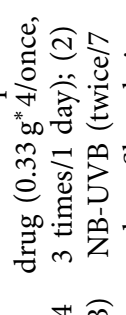 & 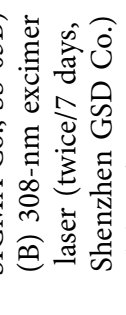 & 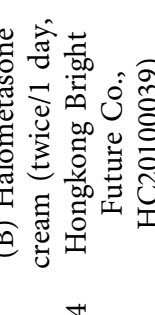 & 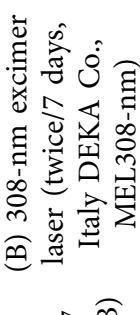 & 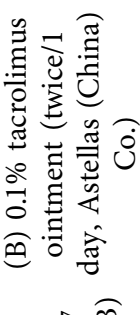 & 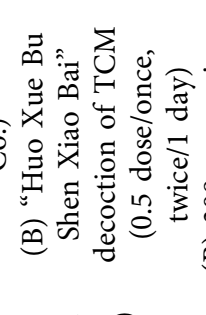 & 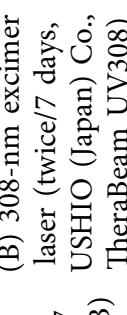 & 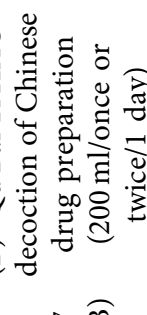 \\
\hline$\stackrel{\vec{\Xi}}{\Xi}$ & 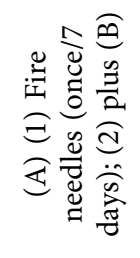 & 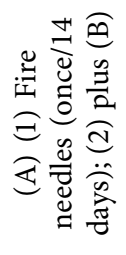 & $\begin{array}{l}\widehat{\varrho} \\
\stackrel{a}{\Xi} \\
\overparen{\Xi}\end{array}$ & 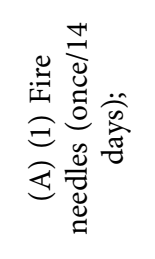 & 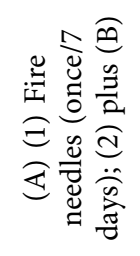 & 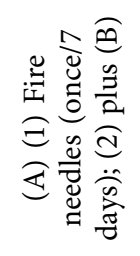 & 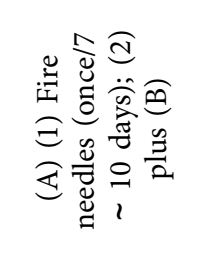 & 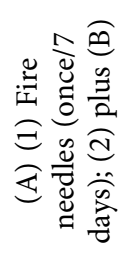 & 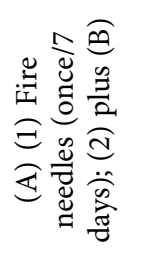 \\
\hline 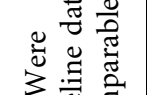 & $\stackrel{\infty}{\approx}$ & $\stackrel{\infty}{\circlearrowright}$ & $\stackrel{\infty}{\circlearrowright}$ & 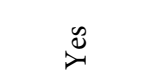 & $\stackrel{\infty}{\approx}$ & $\stackrel{\infty}{\approx}$ & $\stackrel{\infty}{\approx}$ & 号 & $\stackrel{\infty}{\approx}$ \\
\hline 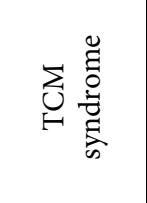 & 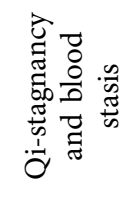 & 岂 & 岁 & 岂 & 凨 & 苔 & 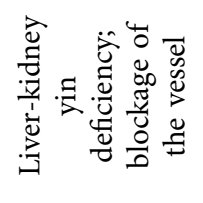 & 觉 & 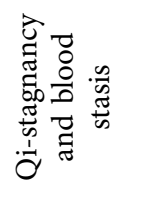 \\
\hline चี & 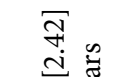 & $\simeq$ & $\simeq$ & $\simeq$ & हैँ & $8 \widetilde{F} \stackrel{\oiiint}{=}$ & $\simeq$ & 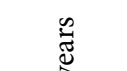 & $\simeq$ \\
\hline 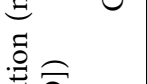 & 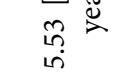 & Z & Z & Z & $\stackrel{n}{n}$ & ஸें & Z & $\stackrel{i n}{i}$ & Z \\
\hline 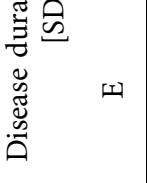 & 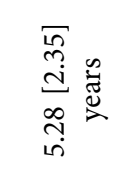 & 岂 & 艺 & 学 & 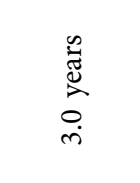 & 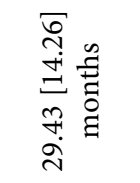 & 号 & 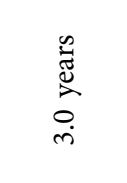 & 学 \\
\hline 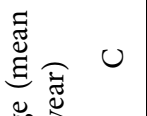 & 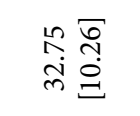 & 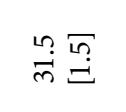 & ñ & 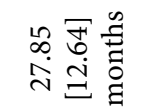 & $\stackrel{\text { an }}{a}$ & 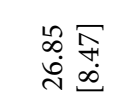 & $\stackrel{m}{m}$ & $\stackrel{0}{\dot{m}}$ & 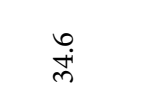 \\
\hline 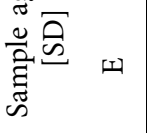 & 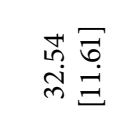 & 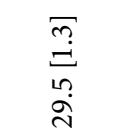 & $\infty$ & 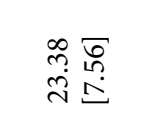 & ?ֶ. & 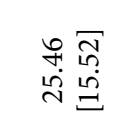 & $\stackrel{m}{m}$ & $\stackrel{n}{m}$ & $\vec{i}$ \\
\hline$\stackrel{0}{2} 0$ & $\ddot{\sim}$ & i & ฉ & $\mathfrak{n}$ & in & $\ddot{m}$ & ঐे & $\stackrel{\infty}{\sim}$ & ले \\
\hline का & $\tilde{\sim}$ & $\widehat{\curvearrowright}$ & ஜ & N & in & $\stackrel{\infty}{m}$ & ลิ & ஓి & mे \\
\hline 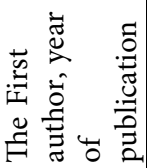 & 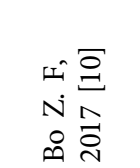 & 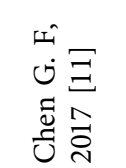 & 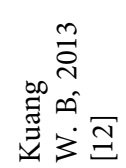 & 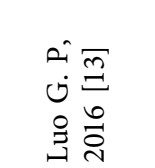 & 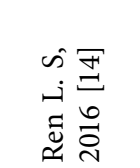 & 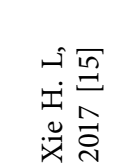 & 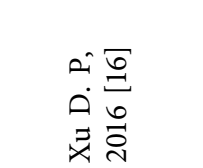 & 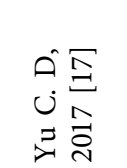 & 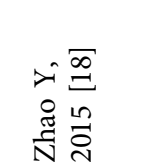 \\
\hline
\end{tabular}




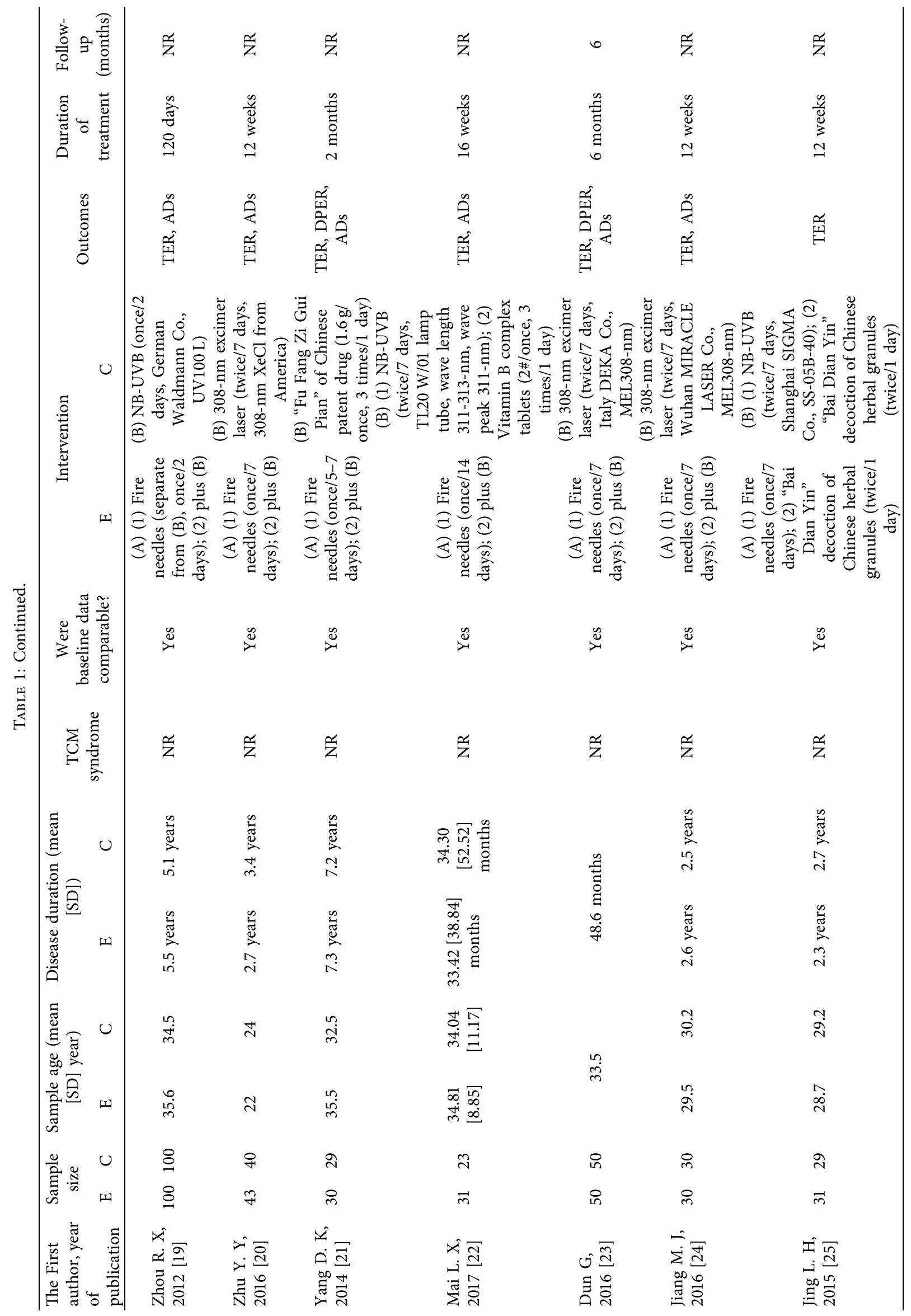




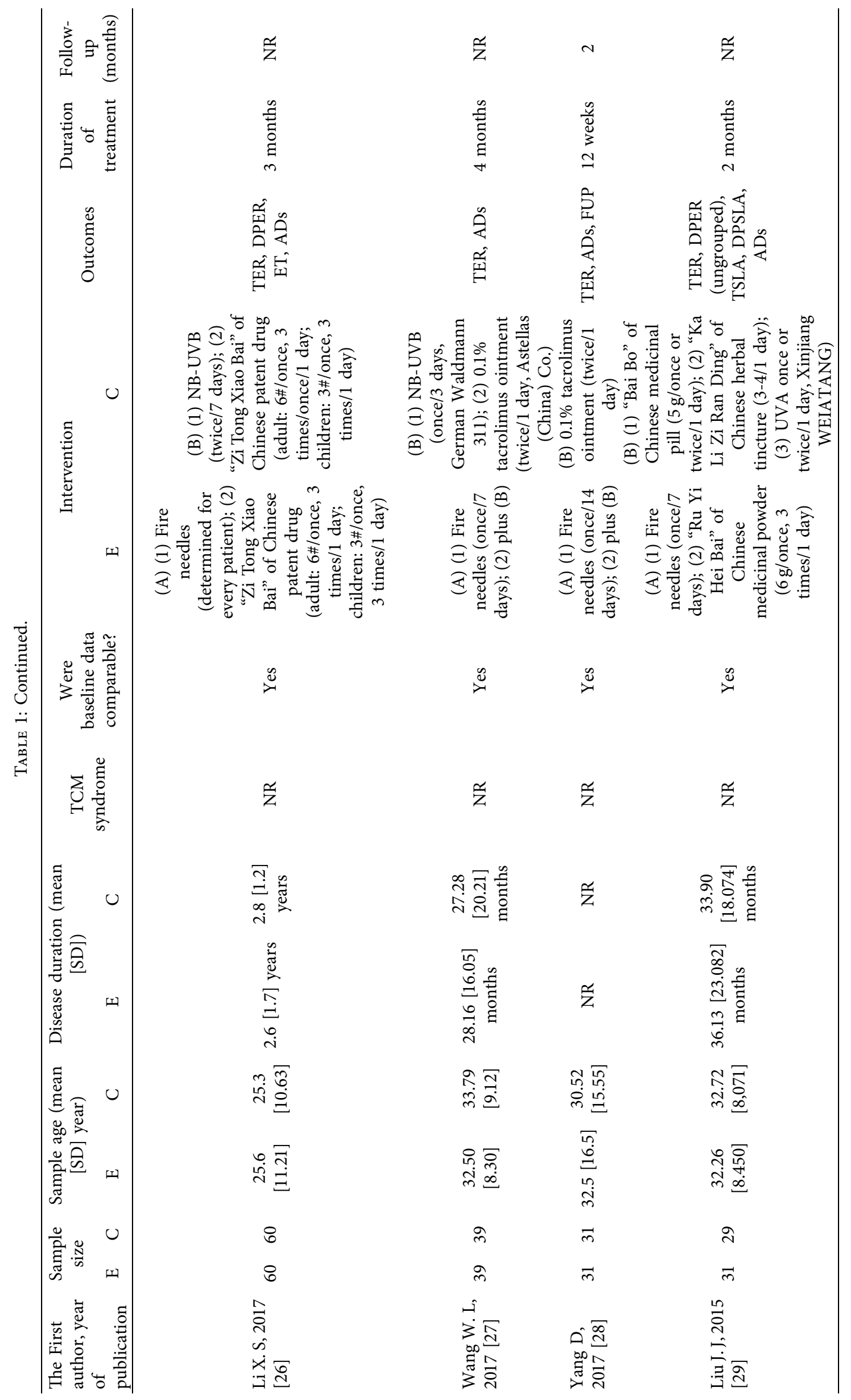




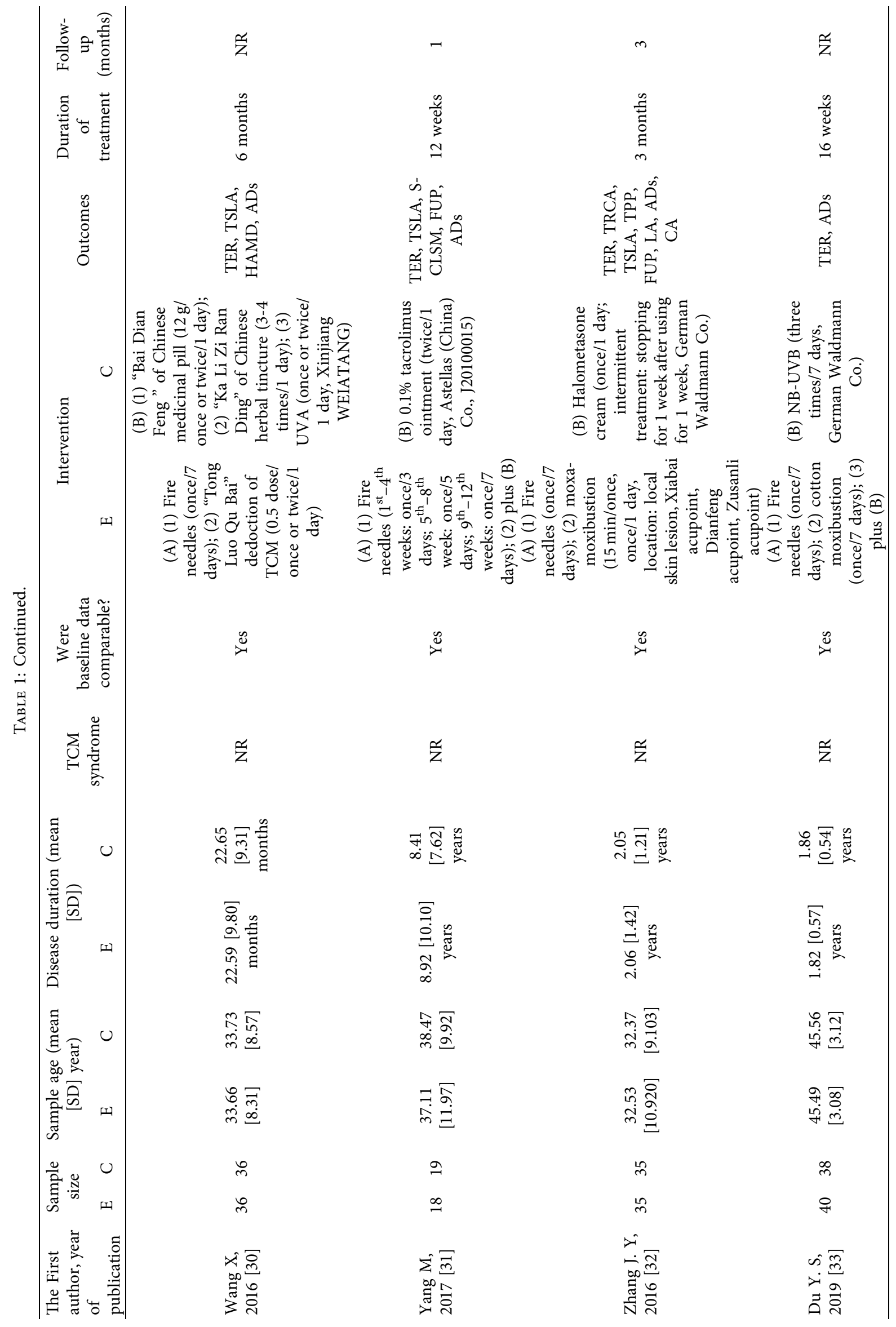




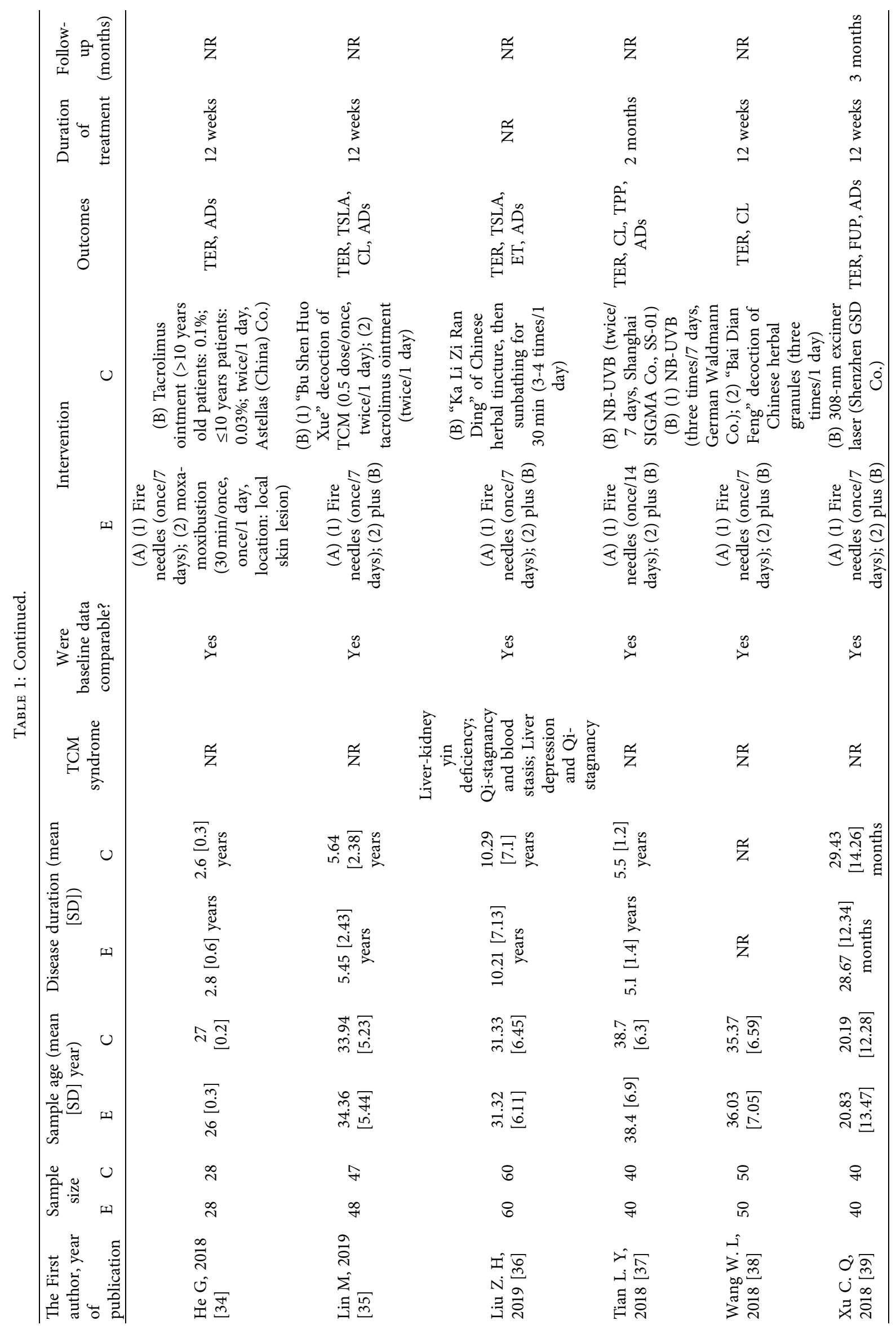




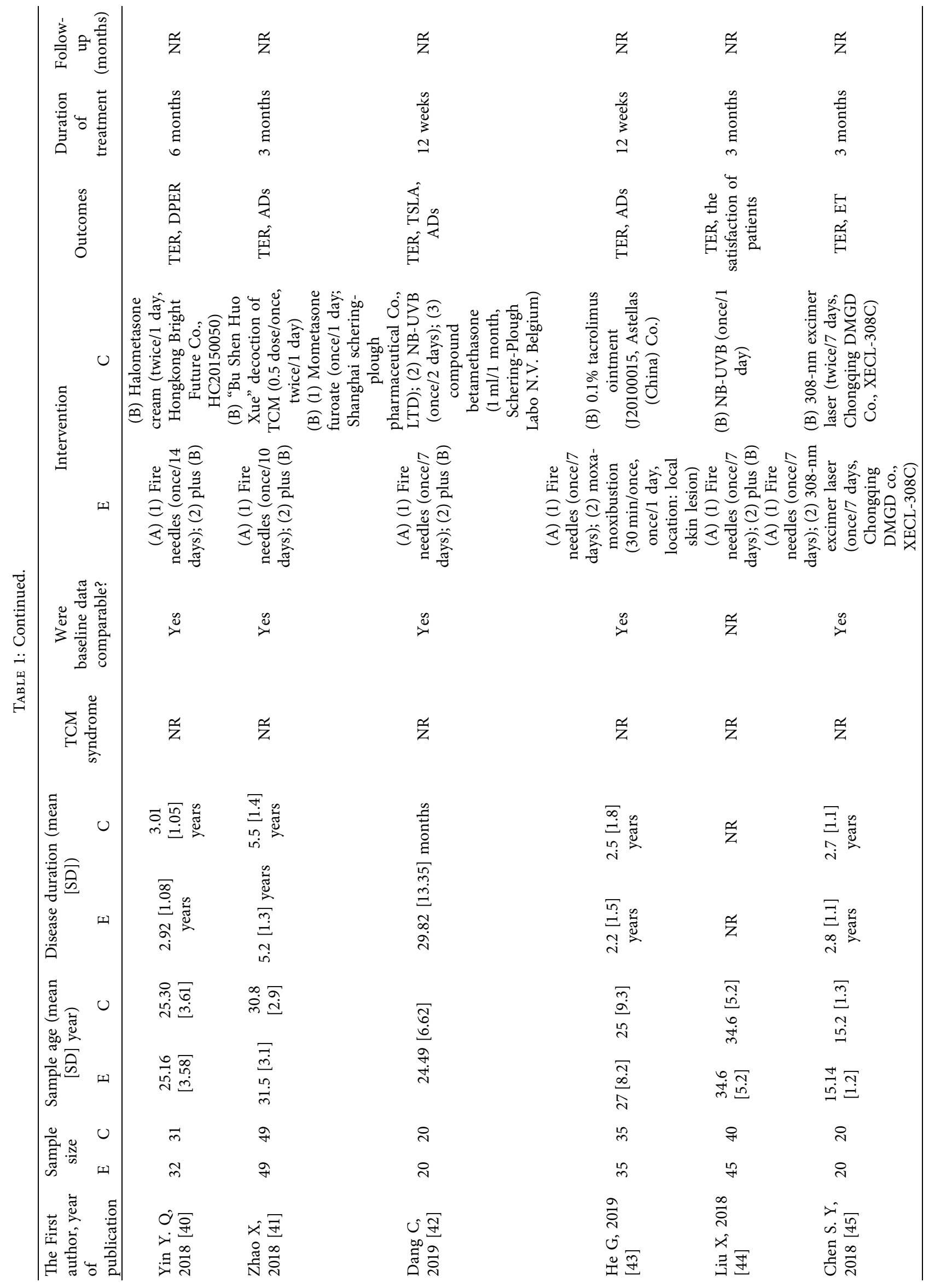




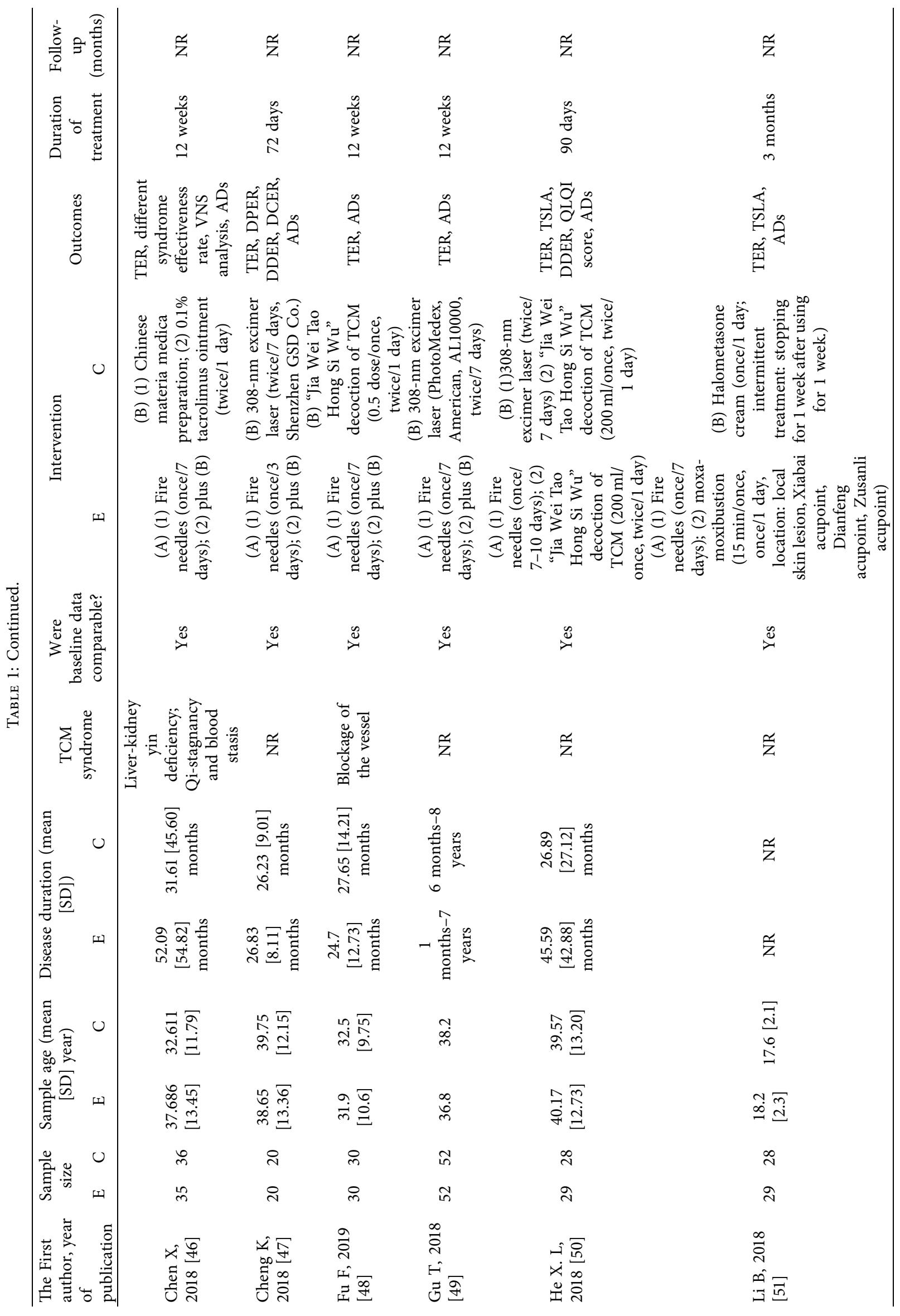




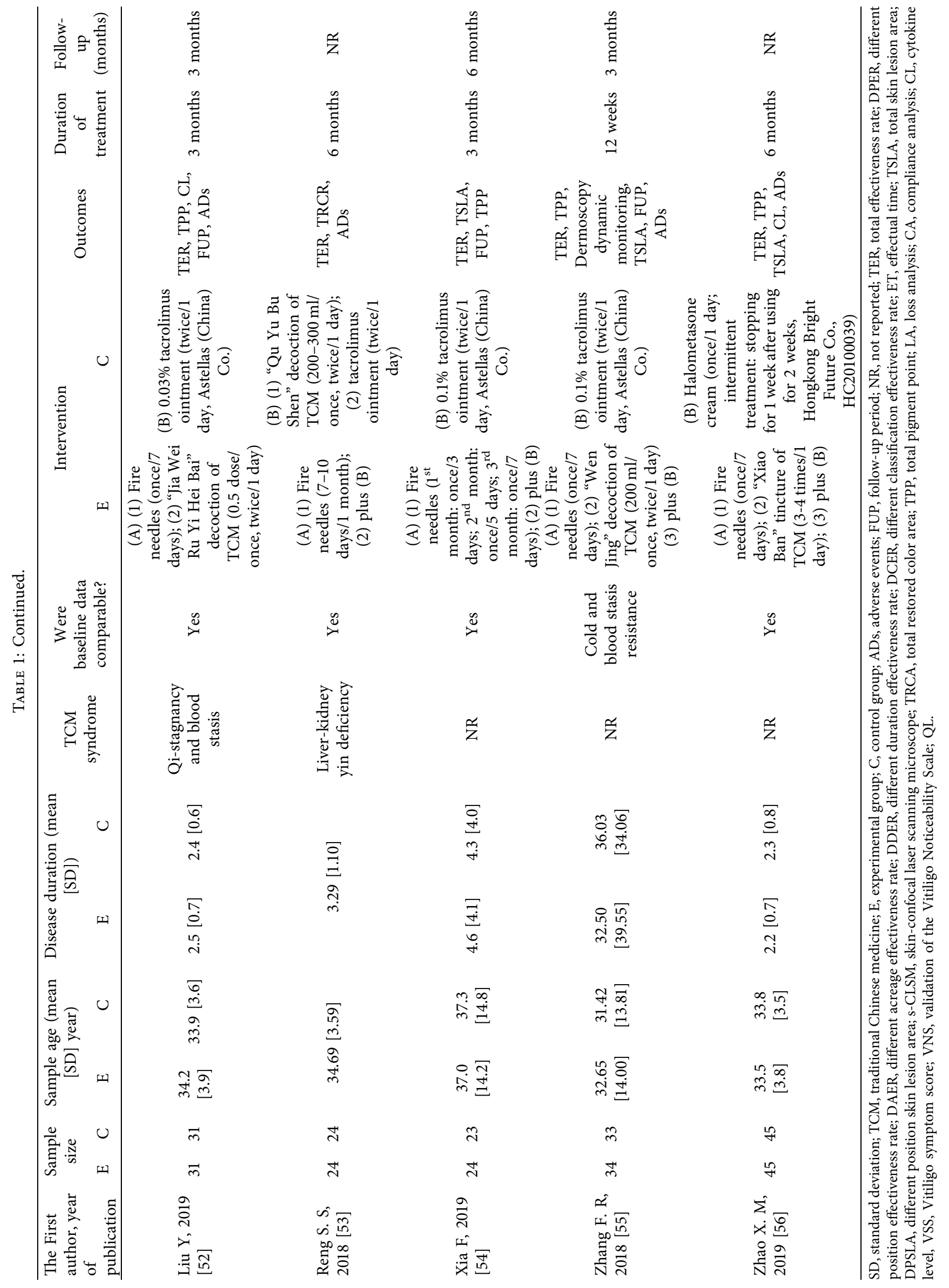




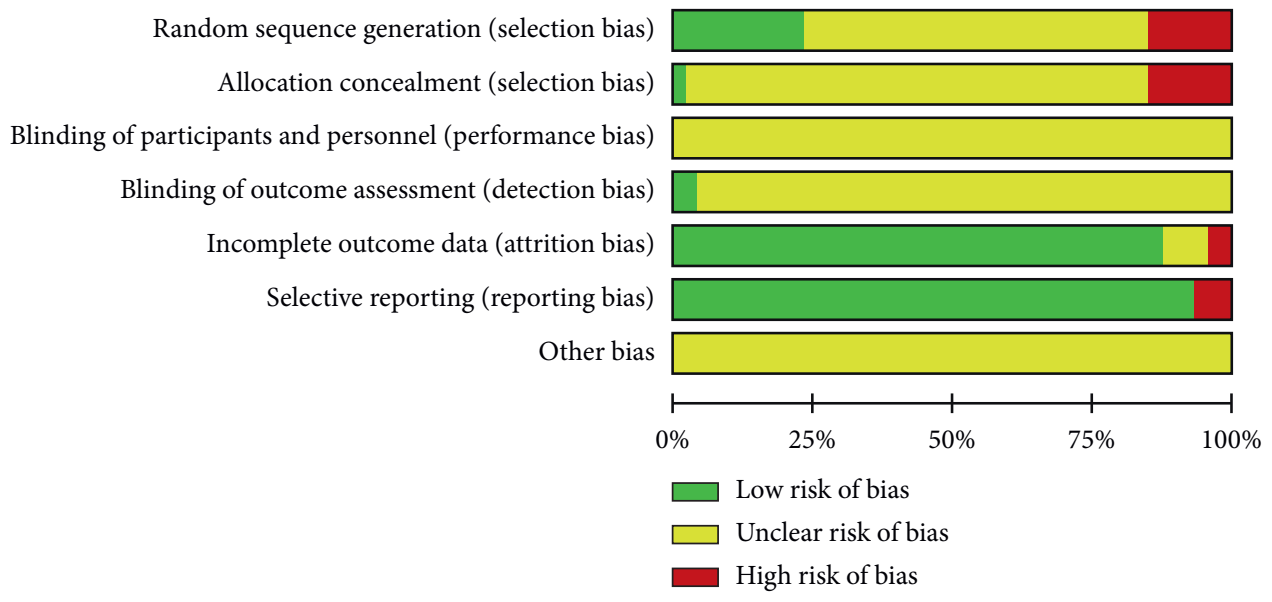

Figure 2: Risk of bias graph.

$[31-34,37,38,43,47,49,54,55]$ used randomization procedures that included random-number tables or the computer randomization method, but only one [32] reported the concealing allocation adequately. Since the interventions involved fire needles, blinding could not be applied to the patients and researchers in all 47 trials; thus, only two trials $[31,32]$ used blinding to assess the outcomes. Four trials $[10,13,19,20]$ did not report the loss of patients to follow-up, while only one trial [22] used an intention-totreat analysis.

\subsection{Meta-Analysis of Primary Outcomes}

3.3.1. Total Effectiveness Rate. Forty-four trials $[10-12,14,16-46,48-56]$ used the number of patients as the unit of statistical outcomes with the total effectiveness rate counted using the NP formula. Of the 44 trials, nine $[12,14,17,20,23,24,39,45,49]$ compared the use of fire needles and a 308-nm excimer laser with a 308-nm excimer laser alone; eight trials $[10,16,18,21,35,36,41,48]$ compared the use of fire needles and other TCM methods with other TCM methods alone; three trials [28, 31, 54] compared the use of fire needles and tacrolimus ointment with tacrolimus ointment alone; 14 trials $[11,19,22,27,33,37,38,40,42,44,46,53,55,56]$ compared the use of fire needles and other treatments with other treatments alone; three $[25,26,50]$ compared the use of fire needles and other TCM methods with both the phototherapy and the same TCM methods; and seven trials $[29,30,32,34,43,51,52]$ compared the use of fire needles and other TCM methods with other traditional treatments.

The subgroup analysis with a fixed-effects model is shown in Table 2(A and B). There was a superior difference in the effect of fire needle therapy with a 308-nm excimer laser $v s$. that of the 308-nm excimer laser alone (RR: 1.46, 95\% CI: $1.32-1.61, P<0.00001)$; fire needle therapy and other TCM methods vs. other TCM methods alone (RR: 1.66, 95\% CI: $1.43-1.94, P<0.00001)$; fire needle therapy and tacrolimus ointment $v s$. tacrolimus ointment alone (RR: 1.57, 95\% CI: $1.15-2.14, P=0.005)$; fire needle therapy and other treatments $v s$. other treatments alone (RR: 1.56, 95\% CI: $1.39-1.74, P<0.00001)$; fire needle therapy and other TCM methods $v s$. both the phototherapy and the same TCM methods (RR: 1.58, 95\% CI: 1.25-2.00, $P=0.0001$ ); and fire needle therapy and other TCM methods $v s$. other traditional treatments (RR: 1.59, 95\% CI: $1.34-1.88, P<0.00001)$. We analyzed the publication bias of the aforementioned trials (Figure 3 ) and found that publication bias existed but was small.

The remaining three trials $[13,15,47]$ used the number of vitiligo skin lesions as the unit of statistical outcomes with the total effectiveness rate counted using the NVSL formula. All three trials involved interventions of fire needle therapy and other treatments vs. other treatments alone (specific types in Table 1 (A), (B)). Using a fixed-effects model (Table 2), we found that there was an obvious difference in the effect of the therapies (RR: 1.41, 95\% CI: 1.24-1.61, $P<0.00001)$.

\subsection{Meta-Analysis of the Secondary Outcomes}

3.4.1. Total Restoration of the Area's Color. Twelve trials [29-32, 35, 36, 42, 50, 51, 54-56] reported data on the total area of the skin lesions both before and after treatment. In a comparison of the restored color on skin lesions using random-effects modeling (Supplementary file 3), we found that treatment including fire needles had a significantly greater effect in restoring the color of the skin than traditional methods without fire needles (MD: 3.40, 95\% CI: 2.11-4.69, $P<0.00001)$.

3.4.2. Total Increased Pigment Point. Six trials $[32,37,52,54-56]$ reported data on the total pigment point of the skin lesions both before and after treatment. In a comparison of the increased pigment point of these skin lesions using random-effects modeling (Supplementary file 4 ), we found that treatment including fire needles had a significantly greater effect in increasing the pigment point of the skin than traditional methods without fire needles (MD: 0.83, 95\% CI: $0.54-1.13, P<0.00001)$. 
TABLE 2: Meta-analysis of the total effectiveness rate in 47 trials.

\begin{tabular}{|c|c|c|c|c|c|c|}
\hline \multirow{2}{*}{ Trial (first author, year of publication) } & \multicolumn{2}{|c|}{ Experimental } & \multicolumn{2}{|c|}{ Control } & \multirow{2}{*}{ Weight (\%) } & \multirow{2}{*}{$\begin{array}{c}\text { Risk ratio } \\
\text { M-H, fixed, 95\% CI }\end{array}$} \\
\hline & Events & Total & Events & Total & & \\
\hline \multicolumn{7}{|c|}{ (A) Trials calculating the total effectiveness rate according to the NP formula } \\
\hline \multicolumn{7}{|c|}{ (1) Fire needle therapy $+308-\mathrm{nm}$ excimer laser versus $308-\mathrm{nm}$ excimer laser alone } \\
\hline Chen S. Y, 2018 & 11 & 20 & 5 & 20 & 0.70 & $2.20[0.93,5.18]$ \\
\hline Dun G, 2016 & 46 & 50 & 34 & 50 & 4.50 & $1.35[1.10,1.66]$ \\
\hline Gu T, 2018 & 41 & 52 & 26 & 52 & 3.40 & $1.58[1.16,2.14]$ \\
\hline Jiang M. J, 2016 & 23 & 30 & 15 & 30 & 2.00 & $1.53[1.02,2.31]$ \\
\hline Kuang W. B, 2013 & 76 & 96 & 44 & 90 & 6.00 & $1.62[1.28,2.05]$ \\
\hline Ren L. S, 2016 & 42 & 50 & 33 & 50 & 4.40 & $1.27[1.01,1.61]$ \\
\hline Xu C. Q, 2018 & 35 & 40 & 20 & 40 & 2.60 & $1.75[1.26,2.44]$ \\
\hline Yu C. D, 2017 & 23 & 30 & 17 & 28 & 2.30 & $1.26[0.88,1.81]$ \\
\hline Zhu Y. Y, 2016 & 36 & 43 & 28 & 40 & 3.80 & $1.20[0.94,1.52]$ \\
\hline Subtotal $(95 \%$ CI $)$ & & 411 & & 400 & 29.80 & $1.46[1.32,1.61]$ \\
\hline Total events & 333 & & 222 & & & \\
\hline \multirow{2}{*}{\multicolumn{7}{|c|}{$\begin{array}{l}\text { Heterogeneity: } \mathrm{Chi}^{2}=8.17, d f=8(P=0.42) ; I^{2}=2 \% \\
\text { Test for overall effect: } Z=7.56(P<0.00001)\end{array}$}} \\
\hline & & & & & & \\
\hline \multicolumn{7}{|c|}{ (2) Fire needle therapy + other TCM methods versus other TCM methods alone } \\
\hline Bo Z. F, 2017 & 12 & 23 & 3 & 23 & 0.40 & $4.00[1.30,12.33]$ \\
\hline Fu F, 2019 & 18 & 30 & 8 & 30 & 1.10 & $2.25[1.16,4.36]$ \\
\hline Lin M, 2019 & 31 & 48 & 15 & 47 & 2.00 & $2.02[1.27,3.23]$ \\
\hline Liu Z. H, 2019 & 45 & 60 & 34 & 60 & 4.50 & $1.32[1.02,1.73]$ \\
\hline Xu D. P, 2016 & 20 & 29 & 11 & 29 & 1.50 & $1.82[1.07,3.08]$ \\
\hline Yang D. K, 2014 & 21 & 30 & 12 & 29 & 1.60 & $1.69[1.03,2.77]$ \\
\hline Zhao X, 2018 & 35 & 49 & 27 & 49 & 3.60 & $1.30[0.95,1.76]$ \\
\hline Zhao Y, 2015 & 27 & 39 & 15 & 39 & 2.00 & $1.80[1.15,2.82]$ \\
\hline Subtotal $(95 \%$ CI $)$ & & 308 & & 306 & 16.60 & $1.66[1.43,1.94]$ \\
\hline Total events & 209 & & 125 & & & \\
\hline \multirow{2}{*}{\multicolumn{7}{|c|}{$\begin{array}{l}\text { Heterogeneity: } \mathrm{Chi}^{2}=9.39, d f=7(P=0.23) ; I^{2}=25 \% \\
\text { Test for overall effect: } Z=6.51 \quad(P<0.00001)\end{array}$}} \\
\hline & & & & & & \\
\hline \multicolumn{7}{|c|}{ (3) fire needle therapy + tacrolimus ointment versus tacrolimus ointment alone } \\
\hline Xia F, 2019 & 20 & 24 & 13 & 23 & 1.80 & $1.47[0.99,2.20]$ \\
\hline Yang D, 2017 & 20 & 31 & 13 & 31 & 1.70 & $1.54[0.94,2.51]$ \\
\hline Yang M, 2017 & 6 & 18 & 3 & 19 & 0.40 & $2.11[0.62,7.20]$ \\
\hline Subtotal $(95 \% \mathrm{CI})$ & & 73 & & 73 & 3.90 & $1.57[1.15,2.14]$ \\
\hline Total events & 46 & & 29 & & & \\
\hline \multirow{2}{*}{\multicolumn{7}{|c|}{$\begin{array}{l}\text { Heterogeneity: } \mathrm{Chi}^{2}=0.32, d f=2(P=0.85) ; I^{2}=0 \% \\
\text { Test for overall effect: } Z=2.81 \quad(P=0.005)\end{array}$}} \\
\hline & & & & & & \\
\hline \multicolumn{7}{|c|}{ (4) Fire needle therapy + other treatments versus other treatments alone } \\
\hline Chen G. F, 2017 & 20 & 27 & 9 & 26 & 1.20 & $2.14[1.21,3.80]$ \\
\hline Chen X, 2018 & 9 & 33 & 12 & 36 & 1.50 & $0.82[0.40,1.69]$ \\
\hline Dang C, 2019 & 15 & 20 & 9 & 20 & 1.20 & $1.67[0.96,2.88]$ \\
\hline Du Y. S, 2019 & 19 & 40 & 15 & 38 & 2.00 & $1.20[0.72,2.01]$ \\
\hline Liu X, 2018 & 29 & 45 & 17 & 40 & 2.40 & $1.52[1.00,2.31]$ \\
\hline Mai L. X, 2017 & 20 & 31 & 5 & 23 & 0.80 & $2.97[1.31,6.73]$ \\
\hline Reng S. S, 2018 & 22 & 24 & 16 & 24 & 2.10 & $1.38[1.01,1.87]$ \\
\hline Tian L. Y, 2018 & 22 & 40 & 13 & 40 & 1.70 & $1.69[1.00,2.87]$ \\
\hline Wang W. L, 2017 & 29 & 39 & 16 & 39 & 2.10 & $1.81[1.19,2.76]$ \\
\hline Wang W. L, 2018 & 45 & 50 & 32 & 50 & 4.20 & $1.41[1.12,1.77]$ \\
\hline Yin Y. Q, 2018 & 18 & 32 & 10 & 31 & 1.30 & $1.74[0.96,3.16]$ \\
\hline Zhang F. R, 2018 & 17 & 34 & 13 & 33 & 1.70 & $1.27[0.74,2.18]$ \\
\hline Zhao X. M, 2019 & 29 & 45 & 20 & 45 & 2.60 & $1.45[0.98,2.15]$ \\
\hline Zhou R. X, 2012 & 68 & 100 & 40 & 100 & 5.30 & $1.70[1.29,2.24]$ \\
\hline Subtotal $(95 \% \mathrm{CI})$ & & 560 & & 545 & 30.30 & $1.56[1.39,1.74]$ \\
\hline Total events & 362 & & 227 & & & \\
\hline \multicolumn{7}{|c|}{$\begin{array}{l}\text { Heterogeneity: } \mathrm{Chi}^{2}=10.84, d f=13(P=0.62) ; I^{2}=0 \% \\
\text { Test for overall effect: } Z=7.54(P<0.00001)\end{array}$} \\
\hline Test for overall effect: $Z=7.54(P<0.00$ & & & & & & \\
\hline \multicolumn{7}{|c|}{ (5) Fire needle therapy + other TCM methods versus phototherapy + same TCM methods } \\
\hline He X. L, 2018 & 26 & 29 & 16 & 28 & 2.20 & $1.57[1.11,2.21]$ \\
\hline Jing L. H, 2015 & 23 & 31 & 13 & 29 & 1.80 & $1.66[1.05,2.61]$ \\
\hline
\end{tabular}


TABLE 2: Continued.

\begin{tabular}{|c|c|c|c|c|c|c|}
\hline \multirow{2}{*}{ Trial (first author, year of publication) } & \multicolumn{2}{|c|}{ Experimental } & \multicolumn{2}{|c|}{ Control } & \multirow{2}{*}{ Weight (\%) } & \multirow{2}{*}{$\begin{array}{c}\text { Risk ratio } \\
\mathrm{M}-\mathrm{H} \text {, fixed, } 95 \% \mathrm{Cl}\end{array}$} \\
\hline & Events & Total & Events & Total & & \\
\hline Li X. S, 2017 & 34 & 60 & 22 & 60 & 2.90 & $1.55[1.04,2.30]$ \\
\hline Subtotal $(95 \% \mathrm{CI})$ & & 120 & & 117 & 6.80 & $1.58[1.25,2.00]$ \\
\hline Total events & 83 & & 51 & & & \\
\hline \multirow{2}{*}{\multicolumn{7}{|c|}{$\begin{array}{l}\text { Heterogeneity: } \mathrm{Chi}^{2}=0.05, d f=2(P=0.97) ; I^{2}=0 \% \\
\text { Test for overall effect: } Z=3.85(P=0.0001)\end{array}$}} \\
\hline & & & & & & \\
\hline \multicolumn{7}{|c|}{ (6) Fire needle therapy + other TCM methods versus other traditional treatments } \\
\hline $\mathrm{He} G, 2018$ & 22 & 28 & 16 & 28 & 2.10 & $1.38[0.95,2.00]$ \\
\hline He G, 2019 & 19 & 35 & 10 & 35 & 1.30 & $1.90[1.04,3.48]$ \\
\hline Li B, 2018 & 22 & 29 & 12 & 28 & 1.60 & $1.77[1.10,2.84]$ \\
\hline Liu J. J, 2015 & 22 & 31 & 14 & 29 & 1.90 & $1.47[0.95,2.28]$ \\
\hline Liu Y, 2019 & 20 & 29 & 13 & 23 & 1.90 & $1.22[0.79,1.88]$ \\
\hline Wang X, 2016 & 24 & 32 & 13 & 33 & 1.70 & $1.90[1.19,3.04]$ \\
\hline Zhang J. Y, 2016 & 24 & 32 & 15 & 33 & 2.00 & $1.65[1.08,2.52]$ \\
\hline Subtotal $(95 \% \mathrm{CI})$ & & 216 & & 209 & 12.50 & $1.59[1.34,1.88]$ \\
\hline Total events & 153 & & 93 & & & \\
\hline \multirow{2}{*}{\multicolumn{7}{|c|}{ Heterogeneity: $\mathrm{Chi}^{2}=3.25, d f=6(P=0.78) ; I^{2}=0 \%$}} \\
\hline & & & & & & \\
\hline Total $(95 \% \mathrm{CI})$ & & 1688 & & 1650 & 100.00 & $1.55[1.46,1.65]$ \\
\hline Total events & 1186 & & 747 & & & \\
\hline \multicolumn{7}{|c|}{ Heterogeneity: $\mathrm{Chi}^{2}=34.54, d f=43(P=0.82) ; I^{2}=0 \%$} \\
\hline \multicolumn{7}{|c|}{ Test for overall effect: $Z=14.34(P<0.00001)$} \\
\hline \multicolumn{7}{|c|}{ Test for subgroup differences: $\mathrm{Chi}^{2}=2.30, d f=5(P=0.81), I^{2}=0 \%$} \\
\hline \multicolumn{7}{|c|}{ (B) Trials calculating the total effectiveness rate according to the NVSL formula } \\
\hline \multicolumn{7}{|c|}{ (1) Fire needle therapy + other treatments versus other treatments alone } \\
\hline Cheng K, 2018 & 19 & 38 & 11 & 34 & 6.60 & $1.55[0.86,2.76]$ \\
\hline Luo G. P, 2016 & 161 & 235 & 118 & 247 & 65.30 & $1.43[1.23,1.68]$ \\
\hline Xie H. L, 2017 & 64 & 115 & 51 & 122 & 28.10 & $1.33[1.02,1.74]$ \\
\hline Subtotal $(95 \% \mathrm{CI})$ & & 388 & & 403 & 100.00 & $1.41[1.24,1.61]$ \\
\hline Total events & 244 & & 180 & & & \\
\hline \multicolumn{7}{|c|}{ Heterogeneity: $\mathrm{Chi}^{2}=0.32, d f=2(P=0.85) ; I^{2}=0 \%$} \\
\hline Test for overall effect: $Z=5.12(P<0.00$ & & & & & & \\
\hline
\end{tabular}

CI, confidence intervals; M-H, Mantel-Haenszel.

3.4.3. Changes in the Cytokine Level. Five trials $[35,37,38,52,56]$ reported various changes in the cytokine level, which demonstrated that fire needle therapy combined with traditional treatments could affect vitiligo-related cytokines. Two trials $[38,56]$ reported the serum interleukin(IL-) 17 level both before and after treatment. In a comparison of changes in the serum IL-17 level using a fixed-effects model (Supplementary file 5), we found that treatment including fire needles had a significantly greater effect on reducing the serum IL-17 level than traditional methods without fire needles (MD: 8.10, 95\% CI: 6.94-9.27, $P<0.00001$ ).

3.4.4. Effectual Time. Three trials $[26,36,45]$ reported the effectual time of treatments in both experimental and control groups. In a comparison of the effectual times using randomeffects modeling (Supplementary file 6), we found that treatment including fire needles had a significantly therapeutic effect in a shorter time than traditional methods without fire needles (MD: $-4.76,95 \% \mathrm{CI}:-7.33$ to $-2.19, P=0.0003$ ).

3.4.5. Rate of the Therapy's Effectiveness in Different Lesion Locations. Three trials [21, 23, 26] examined the effectiveness of treating lesions with different locations in both the experimental and control groups using the NVSL formula. With random-effects modeling, we discovered that interventions including fire needles for lesions placed at limbs had a better effect than interventions that did not use fire needles (RR: 1.60, 95\% CI: 1.31-1.95, $P<0.00001$ ) (Supplementary file 7). At the same time, when the conventional treatments with and without fire needles on either head and neck lesions (RR: 1.13, 95\% CI: 0.78-1.64, $P=0.52$ ) or torso lesions (RR: 1.22 ; 95\% CI: $0.82-1.8$, $P=0.33$ ) were compared, there was no statistically significant difference.

Only one trial [11] examined the effectiveness of treating lesions placed at different locations in both the experimental and control groups using the NP formula, which concluded that treatments ("Bailing pian" of Chinese patent drug and NB-UVB) with fire needles on the head, neck, torso, and limbs had a better effect than without fire needles.

3.4.6. Adverse Effects. Forty-one trials [10, 11, 13-24, 26-37, 39-43, 46-53, 55, 56] mentioned adverse effects; eight trials $[16-18,28,40,49,52,56]$ reported that there was no adverse effect in either the experimental or control groups, and one trial [46] reported adverse effects but did not mention in 


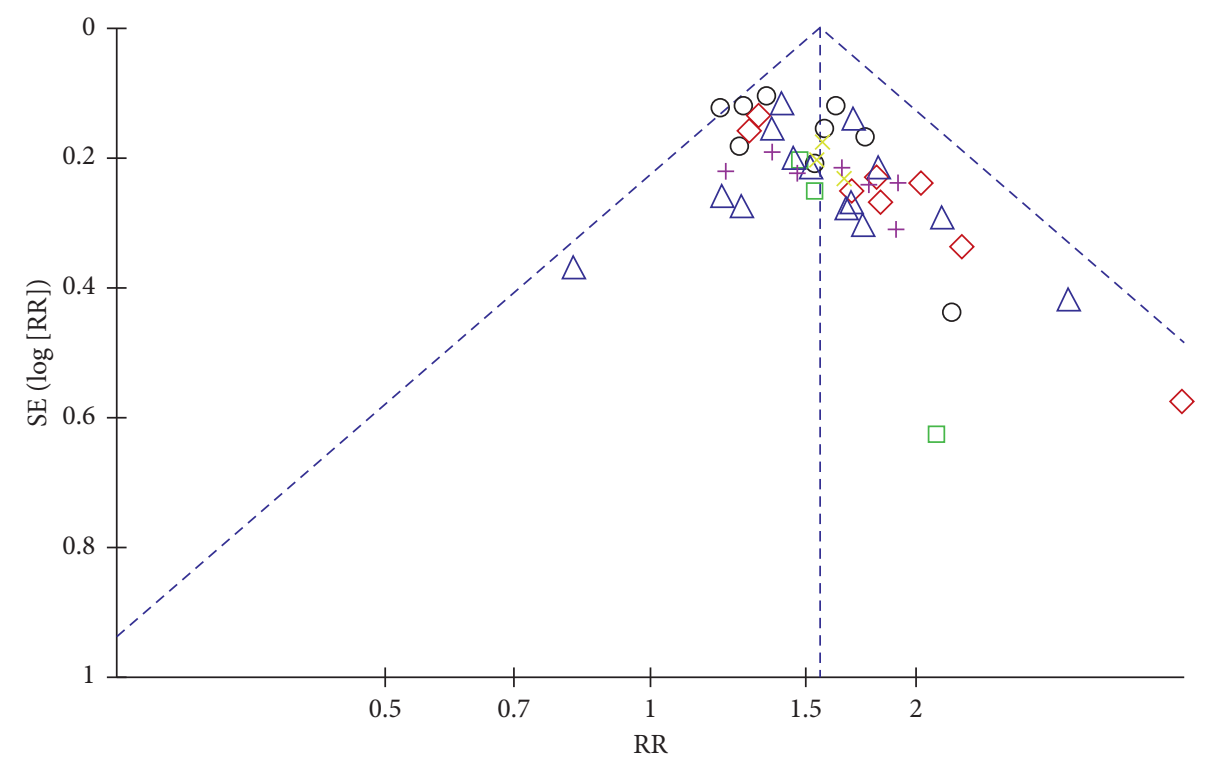
Subgroups
Fire needle $+308 \mathrm{~nm}$ excimer laser versus $308 \mathrm{~nm}$ excimer laser alone
$\diamond$ Fire needle + other TCM methods versus other TCM methods alone
$\square$ Fire needle + tacrolimus ointment versus tacrolimus ointment alone
$\triangle$ Fire needle + other treatments versus other treatments alone
Fire needle + other TCM methods versus phototherapy + same TCM methods
+ Fire needle + other TCM methods versus other traditional treatments

FIgUre 3: Funnel plot of the total effectiveness rate according to the NP formula. TCM, traditional Chinese medicine; NP formula, effectiveness rate $=($ the number of cured patients + the number of markedly effective patients $) /$ the number of total patients $\times 100 \%$.

which groups they occurred. We analyzed the remaining 32 trials with a fixed-effects model (Supplementary file 8) and found that there was no statistically significant difference between the treatments with and without fire needles (RR: 1.15, 95\% CI: $0.89-1.49, P=0.28)$. No serious adverse effects were reported in any of the trials. Mild adverse effects, such as a burning sensation, local redness, and itching of the skin, were tolerable, and they tended to disappear without treatment or after symptomatic treatment (Figure 4).

3.4.7. Recurrence Rates. Eight trials $[14,28,31,32,39$, $52,54,55]$ mentioned the recurrence rate during the followup period. Two trials $[14,31,39,52,55]$ reported that no patients experienced any relapse. With a fixed-effects model, we found that two trials $[28,32,54]$ (Supplementary file 9) showed there was no difference in the recurrence rate between treatments with and without fire needles (RR: 0.90, 95\% CI: 0.17-4.92, $P=0.91$ ).

\section{Discussion}

The data in this study indicated that when compared with traditional methods, fire needles combined with conventional treatments for vitiligo had a higher efficiency, as well as a shorter time before taking effect, and a greater ability to reduce serum cytokines related to vitiligo, restore the color, and increase the pigment point of skin lesions. Limb lesions were more effectively treated when the treatments included fire needles, whereas the effect of treatment on lesions placed at the head, neck, and torso was not significantly different between treatments that used and those that did not use fire needles. There was no statistically significant difference in adverse effects and the recurrence rate during the follow-up period between the methods that included and those that did not include fire needles.

Vitiligo, a disease with a high incidence, is difficult to treat. The main symptoms of skin leukoplakia affect patients' work, daily life, and mental state, thereby leading to serious problems with sleeping and learning. Improvement in the therapeutic outcomes of the condition would improve patients' quality of life [57]. We based this study on the TCM theory, verifying that fire needle therapy, which had some therapeutic effect without serious side effects and could be applied directly to the skin, might be a new way to treat vitiligo.

Chinese medicine proposes that when the human body is greatly stimulated by spirit or material, the "peak potential" of the body's electrical activity is increased, and the electrical activity of the skin damaged by tyrosinase is decreased. The binding of tyrosinase oxidative dopamine with protein is affected, thus destroying the formation of melanosomes. With abnormal neural electrical activity, the function of the cutaneous nerve crest is damaged, along with affected melanocyte formation and metabolism, thereby causing vitiligo [58]. In recent years, fire needle therapy has been proved to 


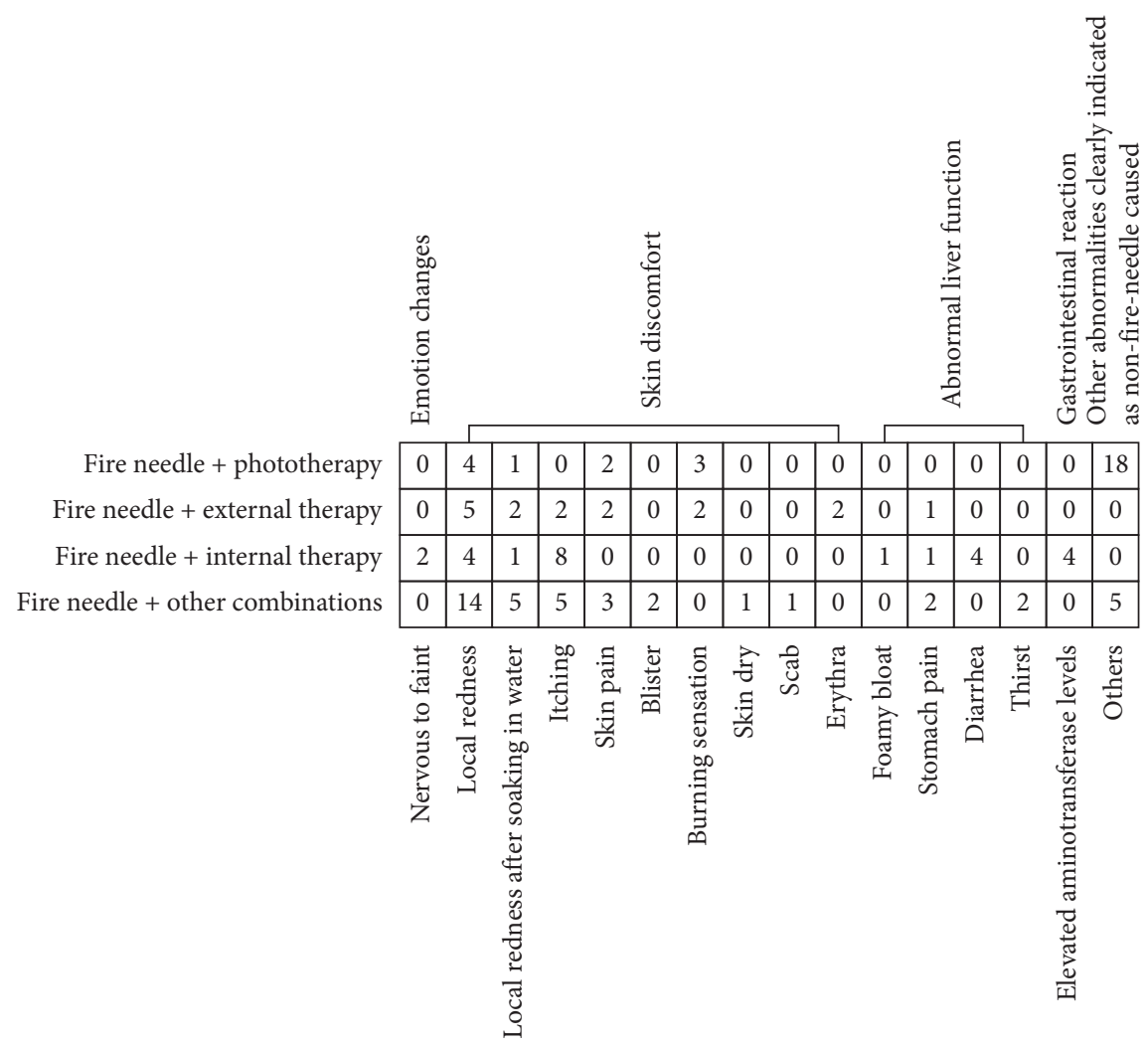

FIgURE 4: Statistics of adverse effects in each experimental group.

be an effective and almost side-effect-free external treatment in TCM [59,60], while clinical research on the application of fire needle therapy for vitiligo has become a popular treatment method. A previous study confirmed that, after the treatment of vitiligo with fire needles, color on the lesions emerged; in the meantime, using a confocal laser scanning microscope, it was observed that dendritic melanocytes appeared in the skin lesions, the pigment content gradually increased and, then, formed a complete pigment ring, and the melanin ring gradually became bright [57]. Laboratory studies also demonstrated that fire needle therapy could improve the recoloration rate of hair follicles and promote the recoloration in refractory locations of acro-limbs. It has been reported that fire needle therapy could help to improve localized blood flow, expand the capillaries, and accelerate the circulation of blood. Nutrition could be strengthened to stimulate the activity of tyrosinase, thus facilitating the formation of melanin [6]. However, acupuncture needling into the skin produces localized inflammatory stimulation that might activate amelanotic melanocytes (AMMC) in the hair root sheath outside the hair follicle, thereby promoting the differentiation of AMMCs to normal melanocytes [61]. The efficacy of fire needle therapy for vitiligo involves the immunomodulatory mechanism that is associated with regulating the proportion of Th17 cells, IL-17, and IL-23 cytokine levels, as well as the function of $\mathrm{T}$ lymphocytes $[62,63]$.

This study had some limitations that should be acknowledged. The studies included in this review generally had poor methodologies, which could have caused bias.
Not all studies mentioned the method of random order generation. In addition, only two trials $[31,32]$ mentioned the blinding method and one trial [32] utilized concealment of allocation. Moreover, the difficulties associated with blinding in studies of acupuncture treatment led to a low methodological quality, causing possible selection bias in RCTs. The safety and efficacy of acupuncture treatment is attracting increasing attention from the domestic and international medical communities. Nevertheless, acupuncture, particularly fire needle therapy, differed significantly in characteristics from drug treatment; thus, effective blinding and the selection of an appropriate placebo had long been recognized as extremely challenging [64]. It was quite clear that the development of more appropriate RCT designs and protocols was an urgent problem that acupuncture therapy faced. Since fire needle therapy was a TCM treatment, all of the studies included were performed in China. The therapy's application in other countries and regions must be studied further. Few studies mentioned the recurrence of lesions and the efficacy of the treatment for lesions placed at different locations, which might have brought about a difference in the results. Additionally, most existing studies had small sample sizes, which might have produced a high level of bias.

The results of this review showed that there is still a lack of well-designed studies on fire needle therapy in the treatment of vitiligo. Since severe adverse effects were not reported in the included studies and the curative effect of therapy with fire needles was definite, we believe that it 
would be worthwhile to conduct further rigorously designed RCTs with larger sample sizes and high methodological quality on fire needle therapy in the treatment for vitiligo in the future.

\section{Conclusions}

Fire needle therapy combined with other conventional treatments had some effect on vitiligo lesions, were not associated with any side-effects, and could be administered in a straightforward manner. More high-quality studies with larger sample sizes should be conducted to make a conclusive judgment of its broader application for treatment.

$\begin{array}{ll}\text { Abbreviations } \\ \text { TCM: } & \text { Traditional Chinese medicine } \\ \text { RCTs: } & \text { Randomized controlled trials } \\ \text { NP } & \text { Effectiveness rate }=(\text { the number of cured } \\ \text { formula: } & \begin{array}{l}\text { patients }+ \text { the number of markedly effective } \\ \text { patients }) / \text { the number of total patients } \times 100 \%\end{array} \\ \text { NVSL } & \text { Effectiveness rate }=(\text { the number of cured } \\ \text { formula: } & \begin{array}{l}\text { vitiligo skin lesions + the number of markedly } \\ \text { effective vitiligo skin lesions }) / \text { the number of }\end{array} \\ & \text { total vitiligo skin lesions } \times 100 \% \\ \text { RRs: } & \text { Risk ratios } \\ \text { CIs: } & \text { Confidence intervals } \\ \text { MDs: } & \text { Mean differences } \\ \text { NB-UVB: } & \text { Narrow-band ultraviolet B light } \\ \text { AMMC: } & \text { Activate amelanotic melanocyte } \\ \text { IL: } & \text { Interleukin } \\ \text { vs: } & \text { Versus. }\end{array}$

\section{Data Availability}

All data generated during this study are included in this article and in the Supplementary Information files.

\section{Conflicts of Interest}

The authors have no conflicts of interest to declare.

\section{Authors' Contributions}

Y.L., X.L., and B.L. were involved in conceptualization. Y.L. and W.Q. were involved in data curation. X.L., Y.L., and W.Q. performed formal analysis. B.L., X.L., and X.Y.S. acquired funding. Y.L., Y.R., and Y.Z. conducted investigation. X.L., T.D., and L.K. were responsible for methodology. X.L., B.L., and Y.L. were involved in project administration. X.L., X.Y.S., and L.L. acquired resources. Y.L. and Q.Z. were responsible for software. B.L. and X.L. were involved in supervision. W.Q., L.K., and H.B.Z. conducted validation. M.X., X.C., and Y.L. were involved in visualization. Y.L. and W.Q. wrote the original draft. Y.L., W.Q., and X.L. were involved in review and editing. Ying Luo and Wei Qian contributed equally to this work.

\section{Acknowledgments}

This project was supported by the NSFC of China (no. 81874470 ). It was also supported by a grant from the National Key Research and Development Program of China (no. 2018YFC1705300), Shanghai Rising-Star Program (no. 16QA1403800), Development Fund for Shanghai Talents (no. 2017047), Young Talent Supporting Program of China Association of Traditional Chinese Medicine (QNRC2-B05), and Shanghai Shen Kang Hospital Development Center Project (no. 16CR2035B).

\section{Supplementary Materials}

Supplementary File 1. National standards of the People's Republic of China- fire acupuncture. Supplementary File 2. Preferred Reporting Items for Systematic Reviews and Metaanalyses checklist. Supplementary File 3. Meta-analysis of total restoration of the area's color. Supplementary File 4. Meta-analysis of the total increased pigment point. Supplementary File 5. Meta-analysis of the reduced serum interleukin-17 level. Supplementary File 6. Meta-analysis of the effectual time. Supplementary File 7. Meta-analysis of the therapy's effectiveness in different lesion locations. Supplementary File 8. Meta-analysis of adverse effects. Supplementary File 9. Meta-analysis of recurrence rates. (Supplementary Materials)

\section{References}

[1] B. Li and D. D. Chen, Dermatovenerology of Integrated Traditional and Western Medicine, pp. 275-277, China Press of Traditional Chinese Medicine, Beijing, China, 2017.

[2] Y. Zhang, J. S. Mooneyan-Ramchurn, N. Zuo, Y. Feng, and S. Xiao, "Vitiligo nonsurgical treatment: a review of latest treatment researches," Dermatologic Therapy, vol. 27, no. 5, pp. 298-303, 2014.

[3] F. F. Shen, Z. S. Min, and M. Y. Xu, "Study on the pathogenesis of vitiligo," Jilin Journal of Traditional Chinese Medicine, vol. 33, pp. 247-249, 2013.

[4] G. R. Lu and A. E. Xu, "Treatment status and progress of vitiligo," Acta Universitatis Medicinalis Anhui, vol. 51, pp. 1713-1716, 2016.

[5] L. X. Du, Y. L. Hou, Y. R. Sun et al., "Research progress of vitiligo treated by western medicine combining with traditional Chinese medicine," Chinese Journal of New Clinical Medicine, vol. 9, pp. 78-82, 2016.

[6] F.-M. Zhao, L.-G. Jiang, and C. He, "A new channel allocation scheme for hierarchical wireless networks," Journal of Shanghai Jiaotong University (Science), vol. 13, no. 1, pp. 40-45, 2008.

[7] T. Wang, Y. Ren, D. Di, Q Zhu, and G Luo, "Segmental vitiligo treated by fire needle therapy: a case series," European Journal of Dermatology: EJD, vol. 28, no. 1, pp. 118-119, 2018.

[8] L. L. Sun, H. X. Liu, Z. Z. Wang et al., "Observation on theapeutic effect of filiform fire needle assisted $308 \mathrm{~nm}$ excimer laser on vitiligo of different parts," Chinese Acupuncture, vol. 39, pp. 936-939, 2019.

[9] J. P. T. Higgins and S. Green, Cochrane Handbook for Systematic Reviews of Interventions, Cochrane Collaboration Website, London, UK, 2018. 
[10] Z. F. Bo and X. J. Ding, "Clinical observation on fire needles and "Shu Gan Huo Xue" decoction combination for the treatment on 23 cases of vitiligo," Nei Mongol Journal of Traditional Chinese Medicine, vol. 36, pp. 64-65, 2017.

[11] G. F. Chen, S. L. Liao, and J. Y. Guan, "Clinical observation on fire needles and NB-UVB combination for the treatment on 53 cases of stable vitiligo," Nei Mongol Journal of Traditional Chinese Medicine, vol. 36, pp. 193-194, 2017.

[12] W. B. Kuang, "Clinical observation on fire needles and $308 \mathrm{~nm}$ excimer laser combination for the treatment on 186 cases of childhood vitiligo," China Health Care \& Nutrition, vol. 23, pp. 5376-5377, 2013.

[13] G. P. Luo, T. J. Wang, Y. Y. Ren et al., "Efficacy of fire needles combined with halometasone cream in the treatment of vitiligo," Diagnostic Therapy Journal of Dermatology-Venereology, vol. 23, pp. 89-92, 2016.

[14] L. S. Ren, X. H. Fu, H. J. Wang et al., "Efficacy observation of fire needles combined with $308 \mathrm{~nm}$ excimer laser for stable vitiligo," Chinese Medicine Cosmetology, vol. 6, pp. 76-78, 2016.

[15] H. L. Xie, J. L. Zhang, and D. H. Li, "Efficacy observation of fire needles combined with tacrolimu," Guiding Journal of Traditional Chinese Medicine and Pharmacology, vol. 23, pp. 67-69, 2017.

[16] D. P. Xu and Y. Gao, "Clinical observation on the clinical efficacy of invigorating blood and kidney combined with fire needles in treating vitiligo," Health For Everyone, vol. 22, p. 137, 2016.

[17] C. D. Yu and J. Yan, "Curative effect observation and nursing care of milli fire needles combined with $308 \mathrm{~nm}$ excimer laser in treating vitiligo," Clinical Journal of Chinese Medicine, vol. 9, pp. 96-98, 2017.

[18] Y. Zhao, C. Q. Zou, Y. Zhang et al., "Clinical observation on fire needles and Chinese medicine combination for the treatment of blood stasis type of vitiligo," Journal of Guiyang College of Traditional Chinese Medicine, vol. 37, pp. 43-45, 2015.

[19] R. X. Zhou, "Clinical effect observation on fire needles and NB-UVB combination for the treatment on 100 cases of vitiligo," Hebei Journal of Traditional Chinese Medicine, vol. 34, pp. 1850-1851, 2012.

[20] Y. Y. Zhu, S. H. Huang, and Y. J. Chen, "Clinical effect observation on fire needles and $308 \mathrm{~nm}$ excimer laser combination for the treatment on vitiligo," Chinese Medicine and Hygiene, vol. 1, p. 191, 2016.

[21] D. K. Yang and J. Xu, "Clinical observation on fire needles and "Fu Fang Zi Gui Pian" of Chinese patent drug combination for the treatment on vitiligo," Journal of New Chinese Medcine, vol. 46, pp. 167-169, 2014.

[22] L. X. Mai, G. Z. Yang, L. F. Liang et al., "Effect of milli fire needles combined with NB-UVB therapy for stable limited vitiligo," Liaoning Journal of Traditional Chinese Medicine, vol. 44, pp. 1487-1490, 2017.

[23] G. Dun, L. S. Ren, and Z. Z. Bian, "Clinical research on fire needles and $308 \mathrm{~nm}$ excimer laser combination for the treatment on vitiligo," Chinese Journal of Clinical, vol. 10, pp. 81-82, 2016.

[24] M. J. Jiang and X. J. Sun, "Clinical analysis of 30 cases on fire needles and $308 \mathrm{~nm}$ excimer laser combination for the treatment on vitiligo," Nei Mongol Journal of Traditional Chinese Medicine, vol. 35, p. 132, 2016.

[25] L. H. Jing, Y. Qu, Z. L. Liu et al., "Observation on the clinical curative effect of traditional Chinese medicine granule combined with fire needles therapy in the treatment of stable localized vitiligo," Journal of Clinical Medicine Literature, vol. 2, pp. 5264-5265, 2015.

[26] X. S. Li, "Clinical effect of milli-fire needles combined with Zitong Xiaobai tablets in treatment of localized vitiligo: an analysis of 60 cases," Hunan Journal of Traditional Chinese Medicine, vol. 33, pp. 12-15, 2017.

[27] W. L. Wang, F. L. Liu, Y. Chen et al., "Curative effect of milli fire needles, NB-UVB and tacrolimus ointment triple therapy in treatment of vitiligo," Journal of Norman Bethune Medical Sciences, vol. 15, pp. 435-436, 2017.

[28] D. Yang, A. M. Liu, L. F. Yang et al., "Clinical observation of $0.1 \%$ tacrolimus ointment combined with fire needles to treat stable vitiligo," Chinese Journal of Dermatology and Venereology, vol. 39, pp. 451-452, 2017.

[29] J. J. Liu, "Clinical observation of compound Ru Yi Hei Bai San of traditional chinese medicine combined with acupuncture for treating boundedness vitiligo phase of consolidation," MMS. thesis, Heilongjiang University of Chinese Medicine, Harbin, China, 2015.

[30] X. Wang, "The clinical observation of decoction based on the theory of collateral disease combined with fire needles in the treatment of stable vitiligo," MMS. thesis, Heilongjiang University of Chinese Medicine, Harbin, China, 2016.

[31] M. Yang, "Study on multi-center randomized controlled open efficacy of vitiligo treated with milli-fire needles," MMS. thesis, XinJiang Medical University, Ürümqi, China, 2017.

[32] J. Y. Zhang, "The clinical research on the stability limits of vitiligo by fire needles combined with moxibustion," MMS. thesis, Chengdu University of Traditional Chinese Medicine, Chengdu, China, 2016.

[33] Y. S. Du and P. Song, "Clinical observation on the treatment of vitiligo with cotton moxibustion and fire acupuncture combined with NB-UVB," Journal of Practical Traditional Chinese Medicine, vol. 35, pp. 492-493, 2019.

[34] G. He, "Observation on the curative effect of acupuncture and moxibustion on localized vitiligo at stable stage," Chinese Medical Guide, vol. 16, pp. 169-170, 2018.

[35] M. Lin, D. L. Zhang, and Q. C. Diao, "Effects of milled fire needle combined with bushen huoxue recipe in the treatment of common type vitiligo patients in advanced stage and its effects on serum sicam-1 level," World Journal of Traditional Chinese Medicine, vol. 14, pp. 1306-1309, 2019.

[36] Z. H. Liu, C. Y. He, and Q. Y. Yu, "Observation on the curative effect of fire needle combined with compound kali-cumin tincture in the treatment of vitiligo," Journal of Dermatology and Venereology, vol. 41, pp. 229-230, 2019.

[37] L. Y. Tian, "The clinical efficacy and safety of NB-UVB combined with fire needle therapy," Clinical Research of Traditional Chinese Medicine, vol. 10, pp. 117-119, 2018.

[38] W. L. Wang, Y. Chen, F. B. Liu et al., "Effect of milli fire needle, vitiligo granules, and narrow band ultraviolet $\mathrm{B}$ triple therapy on the expression of IL-17, IL-10, and IL-22 in the peripheral blood of patients with vitiligo," International Medical and Health Bulletin, vol. 24, pp. 2245-2251, 2018.

[39] C. Q. Xu, Y. Xiang, T. G. Chen et al., "Efficacy observation of milli-fire needle combined with $308 \mathrm{~nm}$ excimer laser for stable vitiligo," Jiangxi Medical Journal, vol. 53, pp. 1051-1053, 2018.

[40] Y. Q. Yin, "Clinical value of fireneedle combined with halometasone cream in the treatment of vitiligo," Journal of Bethune Medical Science, vol. 16, pp. 419-420, 2018.

[41] X. Zhao, X. J. Wang, and C. L. Liu, "Clinical observation on the effect of activating blood and tonifying kidney combined with fire acupuncture in the treatment of vitiligo," Chinese 
Journal of Clinical Rational Drug Use, vol. 11, pp. 113-114, 2018.

[42] C. Dang, "Clinical observation of quadruple therapy in the treatment of vitiligo," Health Nutrition in China, vol. 29, pp. 60-61, 2019.

[43] G. He, "Observation on the curative effect of treating acicular vitiligo with acupuncture and moxibustion," Journal of Aerospace Medicine, vol. 30, pp. 860-862, 2019.

[44] X. Liu, "To compare the treatment of fire needle combined with NB-UVB and NB-UVB alone clinical effect difference of vitiligo," Clinical Medicine, vol. 6, p. 46, 2018.

[45] S. Y. Chen, "Clinical observation on the treatment of vitiligo of middle school students by combination of fire needle combined with 308 irradiation," China \& Foreign Medical Treatment, vol. 37, pp. 66-67, 2018.

[46] X. Chen, "Dynamic obsercation of repigmentation process of vitiligo treated by milli fire needle combined with drugs via reflectance confocal microscopy," MMS. thesis, Nanjing University of Chinese Medicine, Nanjing, China, 2018.

[47] K. Cheng, "Clinical observation of fire needle combined with $308 \mathrm{~nm}$ excimer light in the treatment of stable vitiligo," MMS. thesis, Shanxi University of Chinese Medicine, Taiyuan, China, 2018.

[48] F. Fu and L. M. Shen, "The clinical observation of fire needle combine with decoction of modified taohongsiwu in the treatment of vitiligo," World Latest Medicine Information (Electronic Version), vol. 19, pp. 133-134, 2019.

[49] T. Gu, "Clinical effect analysis of fire shallow acupuncture and 308 excimer light on treatment of vitiligo," World Latest Medicine Information (Electronic Version), vol. 18, pp. 126129, 2018.

[50] X. L. He, "Clinical observation of fire needle combined with decoction in the treatment of stable vitiligo and impact on luality of life," MMS. thesis, Gansu University of Chinese Medicine, Gansu, China, 2018.

[51] B. Li, Y. Q. Yu, and Q. Y. Liu, "Clinical analysis of applying fire acupuncture combined with moxibustion in treating localized vitiligo with stable period," Journal of Sichuan of Traditional Chinese Medicine, vol. 36, pp. 182-184, 2018.

[52] Y. Liu, S. G. Zhang, J. J. Wang et al., "Effect of Jiawei Ruyi Heibai powder combined fire acupuncture on the levels of IL23, IFN- $\gamma$ and Hcy in peripheral blood of patients with vitiligo," Modern Journal of Integrated Traditional Chinese and Western Medicine, vol. 28, pp. 2301-2304, 2019.

[53] S. S. Reng and R. H. Gao, "Study on the efficacy and safety of fire acupuncture assisted removing stasis and tonifying kidney in the treatment of vitiligo," Cardiovascular Disease Journal of Integrated Traditional Chinese and Western Medicine, vol. 6, pp. 172-173, 2018.

[54] F. Xia, X. W. Han, and G. J. Liu, "Clinical study on 24 patients with stable vitiligo treated with fire needle combined with tacrolimus," Jiangsu Journal of Traditional Chinese Medicine, vol. 51, pp. 70-72, 2019.

[55] F. R. Zhang, "Clinical study on Wenjing decoction combined with fire needle therapy for asthenia-temperature obstructive vitiligo," MMS. thesis, Yunnan University Of Chinese Medicine, Kunming, China, 2018.

[56] X. M. Zhao, T. J. Liu, L. Han et al., "Effect of fire acupuncture combined with self-made xiao ban tincture on the treatment of vitiligo and the effect on the expression of TGF- $\beta$, IL- 17 and IL-25 in serum," Modern Journal of Integrated Traditional Chinese and Western Medicine, vol. 28, pp. 1000-1003, 2019.
[57] Y. Y. Wang, Q. C. Diao, C. Z. Ning et al., "Clinical effect of fire needle in treating vitiligo and its CLSM image characteristics," Chongqing Medicine, vol. 47, pp. 1155-1157, 2018.

[58] C. Niu and H. A. Aisa, "Upregulation of melanogenesis and tyrosinase activity: potential agents for vitiligo," Molecules, vol. 22, no. 8, p. 1303, 2017.

[59] C.-N. Lu and H.-H. E. Chiu, "Lateral epicondylitis successfully treated with fire needle therapy: a case report," Chinese Journal of Integrative Medicine, vol. 18, no. 5, pp. 395-397, 2012.

[60] L. Liu, X. Y. Sun, Y. Lu et al., "Fire needle therapy for the treatment of psoriasis: a quantitative evidence synthesis," Journal of Alternative and Complementary Medicine, pp. 1-44, 2020.

[61] M. Xing, X. Yan, X. Sun et al., "Fire needle therapy for moderate-severe acne: a PRISMA systematic review and meta-analysis of randomized controlled trials," Complementary Therapies in Medicine, vol. 44, pp. 253-260, 2019.

[62] Y. Luo, L. Kuai, N. J. Song et al., "Efficacy and safety of fire needle therapy for nodular prurigo: a quantitative study," Evidence-Based Complementary and Alternative Medicine, vol. 2019, Article ID 8797056, 12 pages, 2019.

[63] B. Q. Chen, F. Qian, Y. F. Yang et al., "Effect of fire needle on serum IFN-, IL-17 and IL-23 levels of vitiligo," Hunan Journal of Traditional Chinese Medicine, vol. 33, pp. 107-109, 2017.

[64] Q. Yu, "Blinding method and placebo selection in acupuncture efficacy evaluation," Chinese Journal of Integrative Medicine on Cardio/Cerebrovascular, vol. 4, pp. 196-198, 2003. 